1 Title: Temperature variability alters the stability and thresholds for collapse of interacting

2 species

3 Authors: Laura E. Dee ${ }^{1 *}$, Daniel Okamtoto ${ }^{2}$, Anna Gårdmark $^{3}$, Jose M. Montoya ${ }^{4}$, and Steve J.

4 Miller $^{5}$

5 Affiliations:

$6 \quad{ }^{1}$ Department of Ecology and Evolutionary Biology, University of Colorado, Boulder, Colorado

780309 USA.

$8 \quad{ }^{2}$ Department of Biological Science, Florida State University, Tallahassee, Florida 32303 USA.

$9{ }^{3}$ Department of Aquatic Resources, Swedish University of Agricultural Sciences, Öregrund,

10 Sweden.

$11{ }^{4}$ Theoretical and Experimental Ecology Station, CNRS and Paul Sabatier University, Moulis,

12 France

$13{ }^{5}$ Environmental Studies Program, University of Colorado, Boulder, Colorado

1480309 USA.

15 *Corresponding Author: Laura.Dee@colorado.edu

16 Keywords: temperature variability, thermal performance curves, predator-prey interactions,

17 climate change, climate variability, climate extremes, stability.

\title{
18 Abstract
}

19 Temperature variability and extremes can have profound impacts on populations and ecological

20 communities. Predicting impacts of thermal variability poses a challenge because it has both

21 direct physiological effects and indirect effects through species interactions. In addition,

22 differences in thermal performance between predators and prey and non-linear averaging of

23 temperature-dependent performance can result in complex and counterintuitive population 
24 dynamics in response to climate change. Yet the combined consequences of these effects remain

25 underexplored. Here, modeling temperature-dependent predator-prey dynamics, we study how

26 changes in temperature variability affect population size, collapse, and stable coexistence of both

27 predator and prey, relative to under constant environments or warming alone. We find that the

28 effects of temperature variation on interacting species can lead to a diversity of outcomes, from

29 predator collapse to stable coexistence, depending on interaction strengths and differences in

30 species' thermal performance. Temperature variability also alters predictions about population

31 collapse - in some cases allowing predators to persist for longer than predicted when considering

32 warming alone, and in others accelerating collapse. To inform management responses that are

33 robust to future climates with increasing temperature variability and extremes, we need to

34 incorporate the consequences of temperature variation in complex ecosystems.

\section{Introduction}

Climate change is altering climate extremes and variability of environmental conditions

38 [1], yet much of the focus on the impacts of climate change on wild populations remains on how

39 shifts in average conditions will affect dynamics and distributions [2,3]. Yet as average

40 temperatures shift, both temperature variability and the frequency of extreme events, such as

41 marine heat waves, are changing [1,4-6]. Changes in temperature variation and extremes can

42 have profound impacts on individuals and populations, as temperature affects their rates of

43 metabolism, consumption, somatic growth, reproduction, and survival [7,8]. These processes

44 underpin the productivity and resilience of populations and ecosystems [9], on which ecosystem

45 services, such as fisheries yields [10], and population resilience depend. While the potential

46 impacts of temperature variability and extremes have been demonstrated, and in some cases pose 
47 a greater risk to species than increases in mean temperature $[7,8,11]$, predicting the importance

48 of temperature variability and extremes for populations, communities, and the ecosystem

49 functions and services they support remains challenging. First, temperature can have both direct

50 and indirect effects that create complex feedbacks and dynamics [12]. Directly, temperature

51 affects species through physiological performance. But temperature can also impact species

52 indirectly, as shown for warming $[13,14]$, by increasing or decreasing important resources or

53 prey species (in turn affecting consumers and predators), changing competitive abilities (altering

54 prey abundance distributions), and altering feeding rates and top-down control (affecting prey)

55 [15-18]. Second, even without shifts in mean temperatures, temperature variability can alter

56 mean vital rates because of non-linear relationships between temperature and processes including

57 growth, reproduction, and mortality (Fig 1; [19]). As a result, the responses of populations to

58 increasing temperature variability and extremes induced by climate change, especially in a

59 community context, remains a key research frontier in marine population dynamics, community

60 ecology, and fisheries science.

61 Directly, temperature variability can impact populations of species in a variety of ways,

62 including by altering the rates of fundamental processes that determine population size,

63 extinction risk, and productivity. Increases in the frequency or duration of exposure to extreme

64 temperatures can induce physiological shock leading to depressed somatic growth or lower

65 survival [20,21]. Temperature variability and extremes above cold or warm events, in contrast to

66 mean temperature conditions, can also alter environmental cues that induce or suppress

67 reproductive cycles leading to skip spawning [22], development [23,24], or hatching [23] that

68 change the number of offspring (i.e. recruitment in fish) or cause reproductive failure. Theory

69 and empirical work shows species' performance responds non-linearly and asymmetrically to 
70 environmental temperature, including intrinsic growth rates and other fitness proxies. This

71 relationship is known as a thermal performance curve (TPC) $[8,25,26]$. Because of this non-

72 linearity, changes in temperature variance can affect demographic rates differently than changes

73 in mean temperature alone, due to non-linear averaging and Jensen's inequality (i.e., that the

74 mean of a concave function is smaller than the concave function of a mean, and vice versa for

75 convex functions, see Fig. 1; put in an ecological context reviewed in [19]). This also means that

76 if species are well-adapted to the mean conditions, such that their thermal optimum is close to

77 the average environmental temperature (i.e., in the concave part of the thermal performance

78 curve), then increases in temperature variation around the mean are predicted to reduce a

79 species' performance (e.g., growth rate) $[20,27,28]$. These impacts scale from individuals to

80 population growth rates [29,30]. Similarly, nonlinear TPCs suggest that higher frequency or

81 duration of exposure to temperature extremes, rather than longer-term temperature averages,

82 shape growth and mortality [31] due to species' asymmetric responses to higher temperatures

83 and extremes [20]. Collectively, these nonlinear responses and intermittent exposures to thermal

84 extremes suggest that temperature variability can alter population dynamics in complex ways not

85 explained by warming of average temperatures alone [7,30,32].

86 Simultaneously, temperature can indirectly affect populations through species interactions,

87 thus community and ecosystem responses to temperature variability are complex to predict.

88 Species interactions mediate how temperature impacts a population's growth, biomass, and

89 dynamics. Because species that interact can respond differently to temperature [33,34], species-

90 specific thermal performances can alter interspecific interactions and resulting population

91 dynamics. For instance, if temperature increases prey growth while predators net growth increase

92 less (e.g., due to increased metabolism for larger bodied-species,[35,36]), prey can outgrow 
93 predation windows raising survival [37]. This suggests that if prey thrive directly in response to

94 temperature variation when predators are heat suppressed, prey may flourish in response to

95 heatwaves, and vice versa. Understanding the consequences of temperature variability for marine

96 communities therefore requires considering species interactions, because some species benefit

97 from variable temperatures while others lose (Fig. 1).

98 However, the effects of both temperature variability (or extremes) and species

99 interactions are rarely accounted for in studies of how climate change impacts community

100 dynamics and ecosystem functioning. On one hand, lab studies that quantify the impacts of

101 temperature variability on individuals and populations often focus on the direct physiological

102 effects on performance (e.g., [8,30,32]), with few considering both variation and interactions (but

103 see $[7,38,39])$. On the other hand, most experimental and theoretical studies investigating

104 warming effects on species interactions and their indirect effects do so under different levels of

105 average temperature conditions (e.g.,[15,37]), with less emphasis on variability and extremes

106 (but see e.g., [7]). However, when demographic rates depend non-linearly on temperature and

107 interacting species differ in their thermal responses, we anticipate that the net effect of these

108 processes can lead to unexpected outcomes for population dynamics and stability. Indeed, the net

109 effect of temperature variability could be more positive or negative than considering either effect

110 in isolation.

111 In this paper, we examine how different temperature regimes impact the dynamics of

112 interacting predators and prey, with a focus on multiple types of temperature variability,

113 including increases in temperature variability associated with climate change. We theoretically

114 investigate when considering the combined direct and indirect effects of temperature variability

115 ("net effect") alters predictions for population productivity, stability, and trajectories through 
116 time, relative to considering a constant environment and/or warming average temperatures alone.

117 Specifically, we ask:

118 1) How does increasing temperature variability affect population size through time,

119 occurrence of collapse, and stable coexistence of both predator and prey, relative to a

$120 \quad$ constant environment?

121 2) How do these effects depend on the predator's and prey's TPCs relative to the

122 temperature variability regime, and relative to one another?

123 3) What are the net versus direct effects of temperature variability on these properties when

$124 \quad$ species interact, and when are direct versus net effects acting in opposite directions?

125 4) How do effects of temperature variability compare to and/or modify the effects of $126 \quad$ increasing mean temperatures?

127 We hypothesize that the extent to which the effects of temperature variability shift predictions

128 about population growth, size, and stable coexistence beyond warming average conditions will

129 depend on the 1) thermal variability regime, 2) strength in species interactions, and 3) overlap in

130 TPCs of interacting species (Fig. 1). We motivate our theoretical investigation with a marine

131 predator-prey system, though the model applies more generally to predator-prey systems

132 experiencing both temperature variability and rising temperatures.

\section{Methods}

\section{Model}

135 We model a predator-prey system in which a subset of key parameters depend upon

136 temperature. In line with prior investigations of how temperature affects community dynamics

137 [40-43], we use a Rosenzweig-MacArthur model with a prey population $x(t)$ and predator

138 population $y(t)$ changing as a function of time $t$ : 


$$
\begin{aligned}
& \frac{d x(t)}{d t}=r(T(t)) x(t)\left(1-\frac{x(t)}{K}\right)-y(t) f(x(t), y(t), T(t)), \\
& \frac{d y(t)}{d t}=(c f(x(t), y(t), T(t))-m) y(t) .
\end{aligned}
$$

141 Here, $r$ is the temperature-dependent intrinsic growth rate of the prey population, $K$ their

142 carrying capacity, $T(t)$ is temperature at time $t, m$ the density-independent mortality in the

143 predator, and $f$ denotes the Holling type II functional response of the predator:

$$
f(x(t), y(t), T(t))=\frac{a(T(t)) x(t)}{a(T(t)) h x(t)+1}
$$

145 Here $a$ denotes attack rate, which depends on temperature; $h$ is handling time; and $c$ is conversion efficiency. equilibria: joint extinction, extinction of the predator with the prey at carrying capacity, and a coexistence equilibrium. The coexistence equilibrium is defined by:

$$
x^{*}=\frac{m}{a(c-m h)}, y^{*}=\frac{r}{a}\left(1-\frac{x^{*}}{K}\right)\left(a h x^{*}+1\right)
$$

151 which requires that $a K(c-m h)>m$. We use this equilibrium to set the starting conditions, in

152 terms of the populations levels (abundances), to provide consistent initial conditions under which

153 there are no transitory dynamics in the absence of temperature-dependence.

154 We focus on understanding how temperature variability influences population dynamics

155 via impacts to the predator and the prey species. Particularly, we model how both the attack rate

156 (a) of the predator [44] and the intrinsic growth $(r)$ of the prey [8] depend on temperature.

157 Following current theory and empirical studies, we assume $r(T)$ and $a(T)$ follow unimodal non-

158 linear relationships, with maxima at $T_{r}^{*}$ and $T_{a}^{*}$, respectively (Fig. 1). Although the shape of

159 thermal performance curves (TPCs) will vary among species, populations, and life stages, TPCs

160 are generally considered to have a steep, negative drop-off in performance above optimal 
161 temperatures, but rapid gains in performance at lower temperatures (Fig. 1) [33,45,46]. In our

162 model, we use species-specific asymmetric hump-shaped TPC following Gårdmark et al. (in

163 prep) and [47,48]; see SI for details.

164 We focus on the temperature-dependence of these two parameters, attack rate $a$ and prey

165 growth rate $r$, but recognize that temperature also could influence other parameters in our model

166 (e.g., carrying capacity $K$, handling time $h$, and mortality rate $m$ ) [44,49]. We choose to examine

167 the temperature dependence of $a$ and $r$ because these are the parameters for which we have the

168 most unequivocal information (see [50]). For example, carrying capacity may also vary with

169 temperature [49], but that parameter is not mechanistic in the Rosenzweig-MacArthur model.

170 That is, $K$ may be determined by many factors including primary productivity, nutrient supply,

171 and habitat availability, and temperature may affect each of these in different and complex ways

172 that are more likely linked to exogenous factors that this model does not incorporate.

174 Simulations

175 The way in which temperature variability affects growth and attack rates depends on the

176 distribution of temperatures to which species are exposed and the species' TPCs. Because TPCs

177 for a prey and its predator are likely to differ (Fig. 1), we explore how overlap of TPCs and their

178 relation to temperature distributions affect population dynamics for both species. Thus, we

179 conduct several simulations that combine and vary both: the temperature regime and how

180 species' TPCs relate to one another. For each simulation, we examine how the temperature

181 regime influences average temperature-dependent demographic rates ( $r$ and $a$ ), long-run

182 population means for both predator and prey, and the equilibrium type (either extinction or

183 coexistence with either fixed densities or cycling dynamics). Here, we define 'long-run' predator 
184 and prey populations based on the last $10 \%$ of the simulated time steps (final 200 out of the 2000

185 time steps), which provides time required to reach a stationary distribution (Fig. S1), if one

186 exists.

\section{Temperature regimes}

189 Scenarios of temperature regimes for means and variability

190 The temperature regimes we consider are (1) temperature variability of different

191 amplitudes, but constant mean temperature ('variability-only'), (2) increasing mean temperature,

192 but no variability ('warming-only'), (3) increasing mean temperatures and constant temperature

193 variability of different amplitudes ('warming-and-constant variability') and (4) both increasing

194 mean temperatures and increasing temperature variability ('warming-and-increasing variability').

195 We generate the temperature variability and warming regimes as follows. In the

196 variability-only regime, temperatures oscillate linearly between a maximum $T_{\text {max }}$ and minimum

$197 T_{\min }$, completing one cycle per time period (see SI) to reflect seasonality. In this scenario,

198 increases in temperature variability do not affect the mean temperature $\bar{T}$. In the warming-only

199 regime, temperatures increase linearly from $T_{\min }$ to $T_{\max }$ over the course of the simulation. To

200 facilitate comparison, all warming-only scenarios share the same $\bar{T}$, such that the variability-only

201 and warming-only regimes have the same overall distribution of temperatures for a given $T_{\min }$

202 and $T_{\max }$. We generate these regimes for different sets of $T_{\min }$ and $T_{\max }$, which we refer to as

203 "amplitudes." Finally, the warming-and-variability scenarios combine the linear warming and

204 linear oscillation effects with different amplitudes, where both warming and variability share a

205 specified amplitude (see SI). 


\section{Scenarios with stochastic events driving temperature variability}

208

209

210

211

212

213

214 allowing for both variance and autocorrelation to increase through time. In both cases, we add

215 these stochastic temperature deviations on top of our baseline scenario of oscillating (seasonal)

216 temperatures - enabling us to focus solely on the two types of variability rather than the 217 combination of variability and warming.

warming, or both, we also simulate temperature variability that is stochastic, to test the

sensitivity of our results. We examine two types of stochastic temperature changes (see SI section 1.2). First, we examine major "events" modeled after El Niño; when such an event occurs, the temperature shifts up during a given year. Second, we examine smoother stochastic variability in the form of a Gaussian process with squared exponential covariance function,

\section{Examining how predator-prey TPC overlap and trophic structure modulates effects of}

\section{temperature}

To investigate the extent that TPC overlap between predator and prey species determines

the impact of temperature on each population and their coexistence, we systematically vary the extent of TPC overlap between predator and prey species for each temperature scenario. For each temperature regime, we consider a range of configurations of species' TPCs, defined by the offset $T^{*}-\bar{T}$ (in degrees Celsius) between each species' optimal temperature and the mean environmental temperature (see Fig. 1). Specifically, we consider cases where the TPCs for the predator and prey have offsets of the same magnitude but opposite sign, so that the location of both TPCs is defined in terms of the prey offset (as in Fig 1). When that offset is zero, the predator and prey TPCs overlap exactly. Table S1 defines parameters for these simulations. 
Finally, we conduct an additional set of analyses in which we introduce a second prey

231 species with different thermal affinities than the first prey. To do so, we use a multispecies

232 Holling type II functional response [51], to maintain the same functional form assumptions as

233 above, in order to isolate the effect of adding an additional prey on stable coexistence. To test the

234 influence of multiple prey species with different TPCs on co-existence of predator and prey

235 species, we shifted the first prey TPC and the predator TPC in opposite directions as before, but

236 fixed the second prey TPC at the environmental mean, to facilitate comparison with the single

237 prey case.

240 To quantify the importance of accounting for species interactions when studying the

241 effects of temperature variability, we contrast the direct and net effects (direct plus indirect) of

242 temperature variability on the predator population. For each combination of temperature regime

243 and species' TPCs, we track the predator population for three simulations, with growth and

244 attack rates set to $(i) r(\bar{T})$ and $a(\bar{T}) ;(\underline{i i)} r(\bar{T})$ and $a(T)$; and $(i i i) r(T)$ and $a(T)$; rates defined

245 either at the actual temperature $T$ or at the mean temperature $\bar{T}$ ignoring variability. Thus,

246 simulation (1) ignores all effects of temperature variability, simulation (2) ignores the effects of

247 variability on prey growth, and simulation (3) examines the effects of temperature variability on

248 both species. We define the net effect of temperature variability as the difference in long-run

249 predator population between simulations (3) and (1), while the direct effect is defined by the

250 difference between simulations (2) and (1).

\section{Results}




\section{Effects of temperature variability on long-run populations}

254 Increases in temperature variability produce either stable coexistence, predator-prey cycles,

255 or extinctions (Fig. 2), depending on the difference in predator and prey TPCs and parameters

256 relating to species interactions (predator conversion efficiency and attack rate) (Figure 1; Figure

257 S1). When TPCs for predator and prey coincide (i.e., are identical), variable temperatures can

258 drive the predator extinct from an equilibrium that is stable under conditions without temperature

259 variability (Figure 2b; Fig S1). For higher parameter values of predator conversion efficiency

260 ( $c=0.3$ in Fig. 2a versus $c=0.1$ in Fig. 2b), however, increasing temperature variability can

261 stabilize the system, causing a shift from cyclic dynamics (limit cycle) to a stable equilibrium

262 point (fixed point) (Fig 2a). The opposite effects can arise when the TPCs for the two species are

263 offset so that the prey's temperature optimum is higher. Specifically, when peak attack rates are

264 high, variability can save the predator from extinction, if the prey has a much higher optimum

265 temperature than the predator, or destabilize an otherwise stable equilibrium by inducing cyclic

266 dynamics when the difference in TPCs between predator and prey is less (Fig. 2c). Even when

267 variability does alter the type of equilibrium that arises, equilibrium population levels can be

268 driven up or down.

269 The presence of a second prey species with different thermal affinities from the first prey

270 increases the parameter space with stable coexistence (Fig. S5). Predator feeding on an

271 alternative prey with TPC at mean temperature stabilizes dynamics and prevents extinction,

272 especially when the original prey has a much lower optimal temperature than its predator

273 (negative prey TPC offsets, cf. Fig. 2 and Fig. S5) and for predators with low conversion

274 efficiency (Fig. S5). 
277 Including indirect effects can qualitatively change the impact of temperature variability on

278 populations (Fig. 3; Fig. S2). The true, 'net', effect on long-run population levels can differ from

279 that suggested by studying the direct effect alone, both in terms of direction of the response (Fig.

280 3) and effects on the stability of predator-prey dynamics (Fig. S2). In other cases, the direct

281 effect of temperature variability may imply an unstable equilibrium, but after accounting for

282 indirect effects, the equilibrium remains stable (Fig. S2).

284 Effects of increased temperature variability versus increased temperature mean

285 The effects of increasing mean temperature on long-run population sizes without shorter-

286 term variability are qualitatively different than those of variability without warming, even for an

287 identical distribution of temperatures experienced during the simulation (Fig. 4). For example,

288 increasing mean temperature on predator and prey with completely overlapping TPCs can drive

289 the predator extinct (Fig. 4, red lines) as higher temperatures eventually lead to extended periods

290 of low attack rates (which asymptote towards 0). In contrast, variability with the same

291 distribution of temperatures can lead to a stable coexistence (Fig. 4, blue lines). When mean

292 temperatures increase and variability is present - whether constant (Fig 4, purple lines) or

293 increasing (Fig 4, orange lines) - the predator population survives for longer than under

294 increasing mean temperature alone.

Accounting for stochastic variation in temperature does not change our qualitative

296 conclusions (Figure S6). While moderate stochastic temperature changes knock population

297 trajectories out of asymptotic convergence to equilibrium levels, they do not qualitatively change

298 the means of the long-run population trajectories in response to temperature (Fig. S6) 


\section{Discussion}

Increases in temperature variability can influence populations of interacting species in

302 ways not predicted by considering increases in mean temperatures or the direct physiological

303 effect of temperature variation alone. First, a focus on mean temperature alone misses the highly

304 non-linear responses of species' demographic rates to changes in temperature [52]. Temperature

305 variability, by directly creating complex nonlinear demographic responses, can alter both the

306 viability and stability of populations in either direction (Fig. 2). Second, the net effect of

307 temperature variability when species interact can 4differ from that predicted by the direct effect

308 of temperature on a single species, and even be of opposite sign (Fig. 3). Thus, ignoring indirect

309 effects and making predictions about population dynamics solely from individual demographic

310 rates (e.g. growth) may create erroneous expectations, somethings in the opposite direction (Fig.

3113 ; Fig. S2). This highlights the importance of considering temperature effects in contexts with

312 both intra- and inter-specific interactions. Third, results depend on the differences in predator

313 and prey TPCs (i.e., if the predator TPC is optimized at a higher, identical, or lower temperature

314 than its prey). Finally, results from our model show ignoring temperature variability could over-

315 predict negative impacts of warming on population and community trajectories. Even under

316 warming temperatures, temperature variability results in periods in which temperatures return to

317 a range under which species can grow (Fig. 4), though our results show that the predator

318 populations may still eventually collapse as warming intensifies (Fig. 4d). These findings have

319 important implications for natural communities as temperature variability is predicted to increase

320 further due to global warming. 
That temperature variability increases species persistence can also be seen in single species

322 models, which provide insights into when this 'rescue effect' occurs. This 'rescue effect'

323 depends on how temperature variability influences temperature-dependent demographic rates for

324 hump-shaped TPCs. In a single-species logistic model with temperature-dependent growth, the

325 prey population at time $t$ depends only on the average growth rate $\bar{r}=\overline{r(T(t))}$ of that time period

326 [53]. Depending on the curvature of the TPC over the range of temperatures that a species

327 experiences, temperature variability can either raise (in convex parts of the TPC) or lower

328 average growth (in concave parts of the TPC), with concomitant change in population levels

329 (Fig. 1; see also $[54,55]$ ). If warming drives the mean temperature above the prey's optimal

330 temperature, then temperature variation will span a convex portion of the species TPC, thereby

331 raising average growth and prolonging population persistence (as seen in Fig. 4).

332 Similar insights can be gleaned about the effects of variability on average rates for

333 interacting predator and prey species. Temperature variability is likely to lower average attack

334 rates when the temperature distribution is centered near the peak of the predator's TPC (Fig. 2

335 when TPC offset is around 0), whereas it results in higher average attack rates at lower

336 temperatures (Fig. 2 when TPC offset is large and positive), such that temperature variation

337 occurs in the convex part of the predator's TPC. Temperature variability in these ranges of the

338 TPC thus promotes predator persistence by resulting in sufficiently high attack rates on average.

339 In fact, simulations using the average predator attack and prey growth rates that arise under

340 variable temperatures yield similar overall patterns of stability and coexistence (see Fig. S3 and

341 SI section S1.3 for details).

342 Importantly, our results demonstrate that understanding how a particular temperature regime

343 affects predator populations requires knowledge of temperature dependencies of both species and 
344 how they interact. The true 'net' effect of temperature variability on predator populations may be

345 positive or negative; moreover, because of indirect effects, such responses may differ in sign

346 from predictions made based on changes in attack rate (or other properties in a single species) in

347 isolation (Fig 3). The potential for indirect effects on equilibrium populations is evident from

348 equation (4): prey levels are inversely related to attack rates, while predator levels are

349 proportional to prey growth rates.

350 The difference in TPCs of interacting species dramatically alters the response of ecological

351 communities to variation in temperature (Fig. $2 \& 3$ ). Large differences in temperature optima

352 from $\bar{T}$ lead to predator extinction, regardless of which species has the higher optimum

353 temperature. If prey have lower temperature optima, predator extinction occurs at even lower

354 levels of temperature variability for the same magnitude of TPC offset, compared to if the

355 predator has the lower temperature optimum. The rapid decline of the TPCs above a species'

356 optimum explains this result. Average growth for the prey (or attack rate for predator) is

357 substantially lower when temperature vary above that species' optimum temperature compared

358 to below (i.e. if the temperature optima is shifted downward rather than upward by the same

359 amount). If the prey has a lower temperature optimum than the predator, high temperature

360 variability results in prey population depletion by increasing predator attack rates much more

361 than prey growth rates, eventually leading to predator extinction. These results are consistent

362 with findings from other contexts showing that imbalances in temperature sensitivities of species

363 metabolism and ingestion have important consequences for community dynamics. For example,

364 for invertebrates, metabolism increases faster than ingestion [56,57], which can lead to

365 starvation, and theoretical analyses indicate that this increases population stability but also the

366 risk of starvation and extinction of predators $[13,40]$. 
368 reveals their different impacts on species coexistence and stability, even when the distribution of

369 temperatures under the two regimes is identical. When temperatures warm, long-run predator

370 populations suffer much more than if the same magnitude of temperature change is experienced

371 in a cyclical fashion (Fig. 4). Warming eventually drives predator attack rates to low levels,

372 leading to population declines from natural mortality and a predator population that is not viable.

373 Prey growth also slows, but with reduced predation, the prey population persists. In contrast,

374 under a variability-only scenario, periods of low attack rates are intermixed with stretches of

375 higher attack rates, such that the predator population can survive. Consistent with that intuition,

376 adding variability to a temperature regime with warming can prolong the existence of the

377 predator population, though in other parameterizations variability can accelerate collapse.

We designed our models to quantify the effects that temperature variability can impose

379 on interacting species. However, these models include several simplifying assumptions. Our

380 models assume only the net population growth rate of prey and the attack rate for predators are

381 affected by temperature, but carrying capacities [58], conversion efficiencies [59], mortalities

$382[60,61]$, and handling times $[44,60,62]$ may also depend on temperature. We also include thermal

383 sensitivity in phenomenological rather than explicitly mechanistic forms. More realistic models

384 (e.g., size-structured, age-structured, or individual-based models) could parse out how

385 maturation, fecundity, mortality, individual growth, and consumption simultaneously respond to

386 thermal regimes in different individuals or size-classes when species interact. For example,

387 accounting for both the temperature-dependent processes underlying body growth and for

388 within-population size structure have proven important for understanding how both consumer

389 populations [35] and food chains [63] vary with temperature. However, while this has been 
390 addressed in studies of warming of mean temperatures [35,63,64], it remains to be done for

391 increasing temperature variability. Both the complex responses to increasing temperature

392 variability and the importance of accounting also for indirect temperature effects via interacting

393 species that we demonstrate suggest that predicting how temperature variability would impact

394 size-structured food webs cannot be done a priori. Our study thus calls for addressing

395 temperature variability effects in food webs with approaches accounting for bioenergetics

396 processes and the size-dependence of species interactions.

We demonstrate that the presence as well as the form of temperature variability influence

398 species persistence and coexistence and interact with the effects of warming mean temperatures.

399 Future work could consider the following additional complexities in predicting the effects of

400 temperature dynamics on interacting species in particular systems. First, thermal variability with

401 autocorrelation (e.g., persistent heatwaves) can induce not only prolonged shifts in growth but

402 also mortality, even leading to mass mortality, for instance from oxidative stress shaped by

403 temperature maxima rather than mean temperature [21]. Mass mortality events may be induced

404 by short-term exposure to high temperature, and the threshold temperature for mortality can

405 decrease with greater exposure duration [65], whereas high-frequency variability can also reduce

406 negative effects, such as coral bleaching [66]. Second, the impacts of these short-term events are

407 likely to depend on the species' generation time, relative to time scale of the perturbation. For

408 instance, short-lived species may suffer high mortalities if exposed to unfavorable climate

409 conditions occurring during its short lifespan, compared to longer-lived organism that may better

410 buffer against short-term events. Third, acclimatization [67,68], or short-term evolutionary

411 responses $[48,69]$ to temperature changes could result in inaccurate predictions from models

412 based on historical observations or experiments that are conditional on the environmental history 
413 or genotypes. Nevertheless, our results show temperature variability can alter predictions

414 compared to accounting for increases in temperature means alone, indicating the need for

415 considering temperature variability shapes population stability, collapse, and coexistence when

416 species interact (Fig. 2; Fig. 4).

417 Finally, climate change in the sea is more than warming, variability, and frequency of

418 extreme temperatures. It also encapsulates changes in dissolved oxygen, $\mathrm{pH}$, surface irradiance,

419 salinity, and circulation dynamics -- biophysical changes that are often correlated with

420 temperature fluctuations (IPCC 2019). We chose to focus on temperature dynamics for several

421 reasons. First, there has been a long history of documenting short and long-term variation in

422 ocean temperature either in situ or using satellite reconstruction. In contrast, for other

423 biophysical changes, such widespread and high-resolution data collection and reconstructions are

424 more isolated, sporadic or only recently developed. Second, temperature impacts on physiology

425 and population dynamics have long been a focus in fisheries ecology. Thus, we focus

426 on variation in temperature to quantify how variability in abiotic stressors can alter dynamics

427 with the acknowledgment that other stressors and variability therein are also central in shaping

428 populations and communities and may exhibit independent and multiplicative stresses on

429 communities in unpredictable ways. Here we show that the inherent complex direct and indirect

430 responses of populations and communities to gradual linear temperature changes that

431 characterizes climate warming versus the non-linear and extreme changes that characterize

432 climate variability is challenging. This challenge requires that, before including additional and

433 important complexities, we deeply understand the interplay of climate warming and variability. 
Climate change is increasing not only mean temperatures but also temperature variability

437 (IPCC 2019). Understanding the consequences of temperature variability for population

438 trajectories and dynamics is critical for anticipating how climate change will affect the

439 productivity and stability of animal communities that support important functions and services.

440 Here we find that shifts in temperature variability can destabilize, stabilize or lead to predator

441 collapses, depending on interaction strengths and differences in thermal performance between

442 predators and prey. Our results also show that impacts on species' growth from concurrent

443 changes in variability with warming can change predictions from considering warming alone.

444 Counterintuitively, temperature variability can help a population that would otherwise go extinct

445 due to warming, when warming negatively impacts population growth, and some forms of

446 variability can offset these effects. In other cases, however, ignoring increases in temperature

447 variability associated with climate change leads to underestimation of predator extinction risks.

$448 \quad$ Our results contribute to a growing understanding of how temperature variation will alter

449 life in the oceans, though the theoretical results extend more generally to other predator-prey

450 systems facing variability. Our findings call for future studies advancing the theory on increasing

451 temperature variation in foodwebs. In particular, we encourage accounting for within-species

452 structure and variation in TPCs, as well as testing this in experimental studies in interacting

453 species (over temperature ranges large enough for the non-linear responses to matter). Moving

454 beyond a focus on mean temperatures alone, to advance our understanding of the consequences

455 of temperature variation in complex ecosystems, can improve our ability to inform management

456 responses that are robust to future climates with increasing variability and extremes. 
459 We thank B. Kendall, D. Bradley, R. Gentry, and C. Costello for discussions that benefited

460 earlier iterations of this research, and the Dee lab and J. Ashander for feedback on this draft. This

461 work was partly supported by the Swedish Research Council (Vetenskapsrådet, grant 201503752

462 to AG), and by the FRAGCLIM Consolidator Grant, funded by the European Research Council

463 under the EU Horizon 2020 research and innovation programme (Grant 726176 to JMM). 


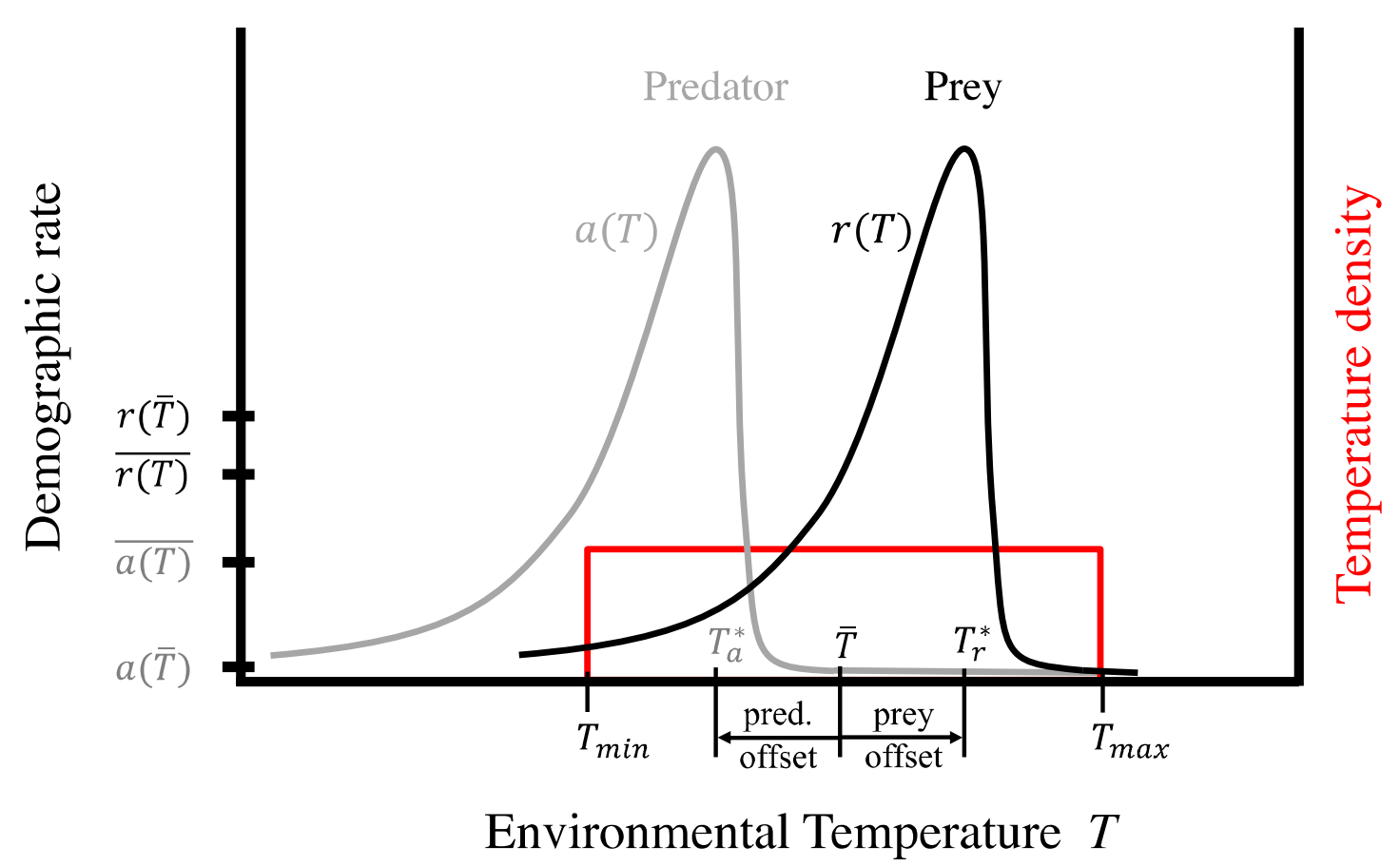

Figure 1. Nonlinear responses to temperature. A conceptual figure of how variable temperatures affect demographic rates, e.g., intrinsic per-capita growth rate $r(T)$ or attack rate $a(T)$, following thermal performance curves (TPCs). Each individual of a species has a

471 temperature optimum $T^{*}$ at which its performance is maximized, which may be offset from the

472 mean environmental temperature $\bar{T}$. When temperature varies, average demographic rates $\overline{r(T)}$

473 and $\overline{a(T)}$ may be higher or lower than demographic rates at the mean temperature $(r(\bar{T})$ and

$474 \mathrm{a}(\bar{T}))$ due to Jensen's inequality. For example, average rates are likely to be smaller for species

475 adapted to their average ecosystem temperature, i.e. $T^{*} \approx \bar{T}$. If the range of temperatures

476 encompasses both convex and concave regions of the TPC, the net effect is indeterminate but 
477 generally nonzero. In experiments, we vary (1) the amplitude of temperature variability $\left(T_{\max }-\right.$

$\left.478 T_{\text {min }}\right)$, and (2) how far the TPCs are offset from the environmental mean temperature $\left(T_{r}^{*}-\bar{T}\right.$

479 and $\left.T_{a}^{*}-\bar{T}\right)$. We restrict offsets to be equal in magnitude but have opposite sign, reporting

480 results in terms of the predator's TPC offset $\left(T_{r}^{*}-\bar{T}\right)$.

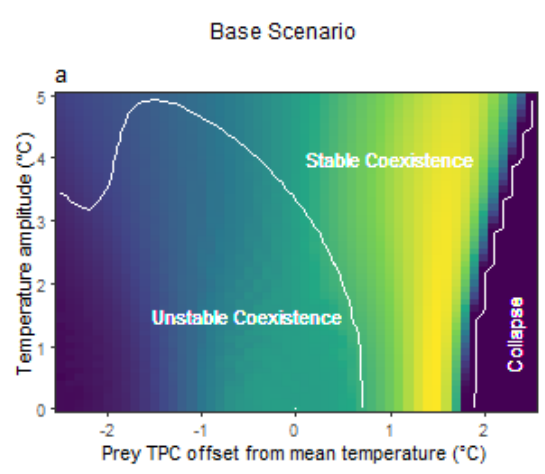

482

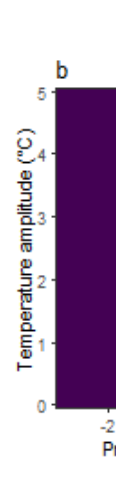

Efficiency $=0.1$

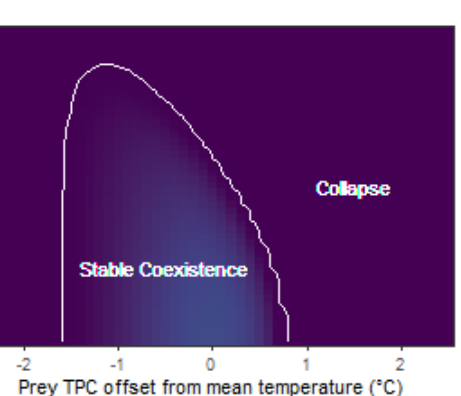

Long-run predator population

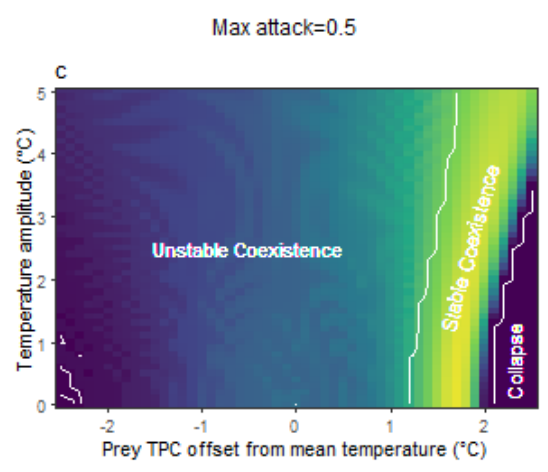

Prey TPC offset from mean temperature $\left({ }^{\circ} \mathrm{C}\right)$

483 Figure 2: Stable predator collapse or predator-prey coexistence depends on both the offset

484 in the predator and prey thermal performance curves (TPCs) and amplitude of

485 temperature variability. Effects of offset in the predator and prey TPCs (x-axis) and amplitude

486 of temperature variability (y-axis) on predator population abundance (colors) and the type of

487 equilibrium that arises: predator collapse or predator-prey coexistence, and whether the latter is

488 stable or unstable (e.g., cycles or oscillatory behavior). Base scenario parameters (a) are

489 mortality $m=0.2$, carrying capacity $K=20$, conversion efficiency $c=0.3$, maximum attack rate

$490 a=0.3$, and handling time $h=0.3$, whereas species interactions are modified in (b-c) by

491 lowering conversion efficiency $c=0.1(\mathrm{~B})$ or increasing attack rate $a=0.5(\mathrm{C})$. TPC parameters

492 are in Table S1. 


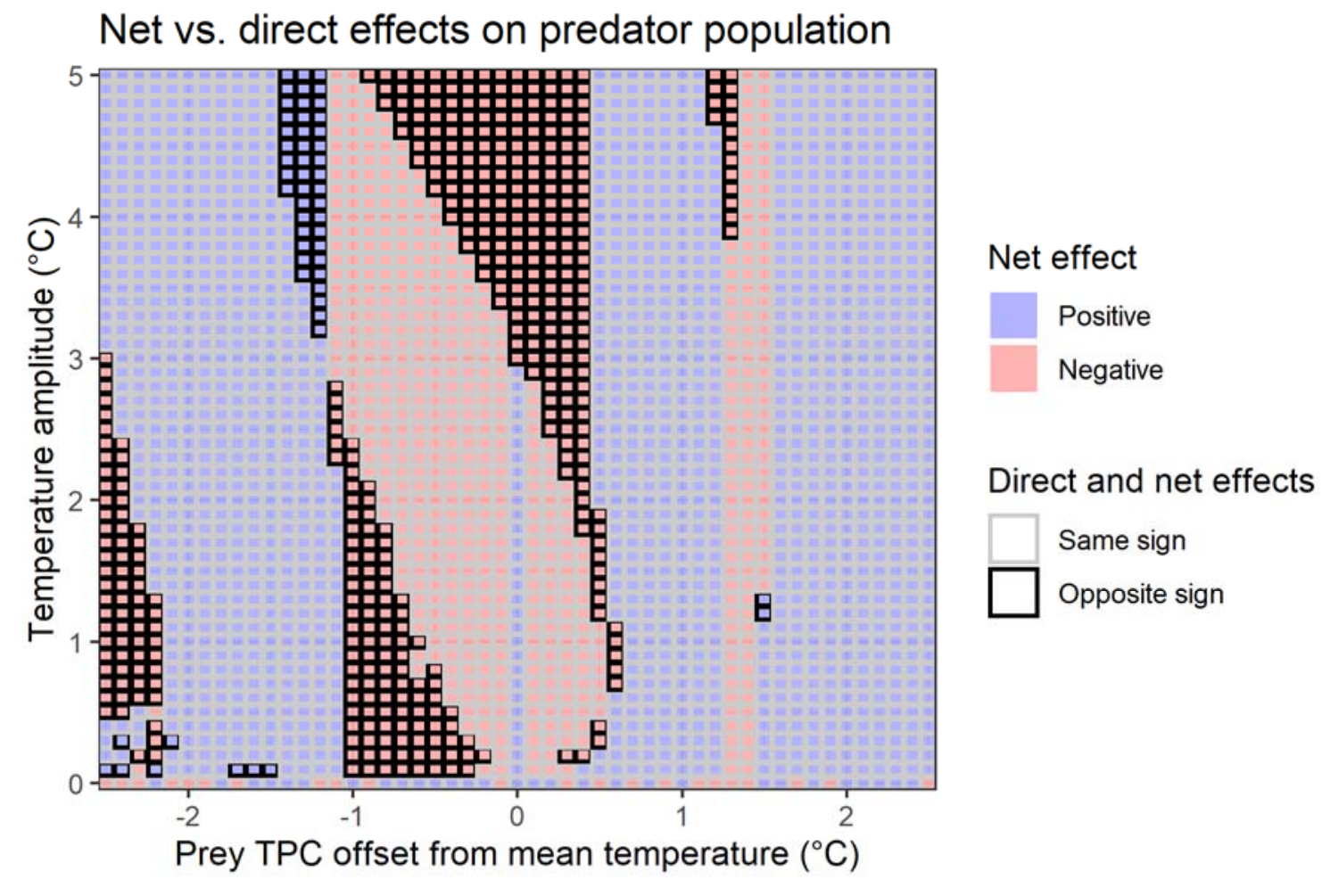

495 Figure 3: The net and direct effects of temperature variability on predator population.

496 Net effects (blue: positive; red: negative) of temperature variability on long-run population levels

497 for the predator, as a function of how far the prey TPC is offset relative to the mean temperature

498 (x-axis) and the amplitude of temperature variability (y-axis). Outlines indicate whether net

499 effects have the same sign (black outline) or not (light gray outline) as when only considering the

500 effects of temperature variability on the predator, ignoring the temperature-dependence of its

501 prey ('direct effects'). For example, red-filled and black-outlined regions indicate

502 parameterizations where considering only temperature effects on the predator would suggest a

503 positive effect of temperature variability for the predator population when the true net effect is

504 negative, due to species interactions. Parameters as in Fig. 2a with TPC parameters in Table S1;

505 see Fig S4 for results from additional parameterizations. 

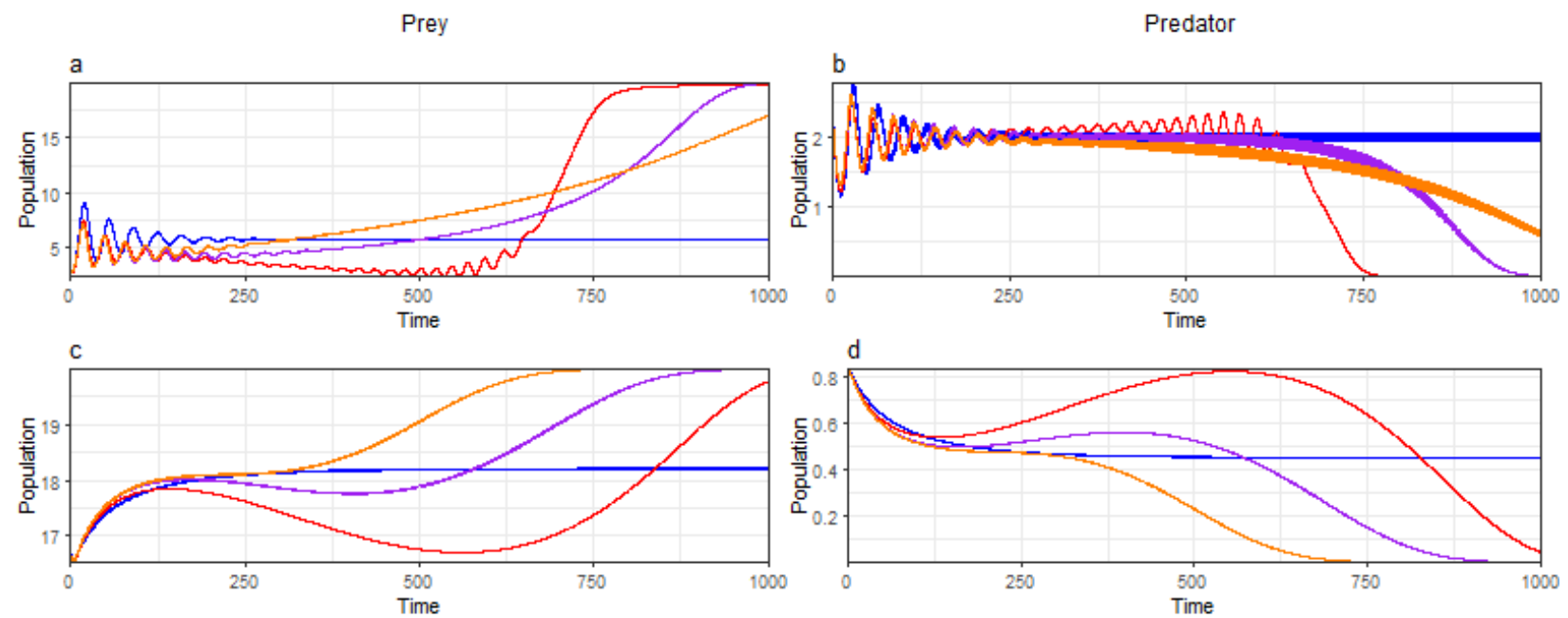

Temperature Scenario - Fixed Variability, No Warming Warming + Fixed Variability

Figure 4. Interacting effects of warming and temperature variability.

509 Population trajectories for the prey $(\mathrm{a}, \mathrm{c})$ and predator $(\mathrm{b}, \mathrm{d})$ under scenarios of warming

510 (increasing mean temperatures) with no variability (red), constant temperature variability with

511 constant mean temperatures (blue), increases in mean temperatures with constant variability

512 (purple), and increases in both mean temperatures and temperatures variability (orange).

513 Variability on top of warming can either delay (panel b) or accelerate (panel d) predator collapse.

514 Panels a and $\mathrm{b}$ reflect model parameters as in Fig $2 \mathrm{a}$ with $T_{\max }-T_{\min }=8^{\circ} \mathrm{C}$; panels c and d

515 reflect model parameters as in Fig $2 \mathrm{~b}$ with $T_{\max }-T_{\min }=2^{\circ} \mathrm{C}$ TPC parameters are in Table S1

516 with zero offset. 


\section{References}

518 1. Easterling, D. R., Meehl, G. A., Parmesan, C., Changnon, S. A., Karl, T. R. \& Mearns, L.

519 O. 2000 Climate Extremes: Observations, Modeling, and Impacts. Science (80-. ). 289,

$520 \quad 2068-2075$.

521 2. Pecl, G. T. et al. 2017 Biodiversity redistribution under climate change: Impacts on

522 ecosystems and human well-being. 9214. (doi:10.1126/science.aai9214)

523 3. Poloczanska, E. S. et al. 2013 Global imprint of climate change on marine life. Nat. Clim.

$524 \quad$ Chang. , 1-7. (doi:10.1038/nclimate1958)

525 4. Cai, W. et al. 2014 Increasing frequency of extreme El Niño events due to greenhouse 526 warming. Nat. Clim. Chang. 4, 111-116. (doi:10.1038/nclimate2100)

527 5. Holbrook, N. J. et al. 2019 A global assessment of marine heatwaves and their drivers.

Nat. Commun. 10, 1-13. (doi:10.1038/s41467-019-10206-z)

529 6. Frölicher, T. L., Fischer, E. M. \& Gruber, N. 2018 Marine heatwaves under global

$530 \quad$ warming. Nature 560, 360-364. (doi:10.1038/s41586-018-0383-9)

531 7. Pansch, C. et al. 2018 Heat waves and their significance for a temperate benthic

532 community: A near-natural experimental approach. Glob. Chang. Biol. 24, 4357-4367.

$533 \quad$ (doi:10.1111/gcb.14282)

534 8. Vasseur, D. A., DeLong, J. P., Gilbert, B., Greig, H. S., Harley, C. D. G., McCann, K. S., 535 Savage, V., Tunney, T. D. \& O'Connor, M. I. 2014 Increased temperature variation poses 536 a greater risk to species than climate warming. Proc. Biol. Sci. 281, 20132612.

$537 \quad$ (doi:10.1098/rspb.2013.2612)

538 9. Svensson, F., Karlsson, E., Gårdmark, A., Olsson, J., Adill, A., Zie, J., Snoeijs, P. \& 539 Eklöf, J. S. 2017 In situ warming strengthens trophic cascades in a coastal food web. 
Oikos 126, 1150-1161. (doi:10.1111/oik.03773)

541 10. Dee, L. E. et al. 2016 Functional diversity of catch mitigates negative effects of

542 temperature variability on fisheries yields. Proc. R. Soc. B 283, 20161435.

$543 \quad$ (doi:http://dx.doi.org/10.1098/rspb.2016.1435)

544 11. Helmuth, B., Russell, B. D., Connell, S. D., Dong, Y., Harley, C., Lima, F. P., Sará, G.,

545 Williams, G. a \& Mieszkowska, N. 2014 Beyond long-term averages: making biological

546 sense of a rapidly changing world. Clim. Chang. Responses 1, 6. (doi:10.1186/s40665-

$547 \quad 014-0006-0)$

548 12. Rijnsdorp, A. D., Peck, M. A., Engelhard, G. H., Möllmann, C. \& Pinnegar, J. K. 2009

549 Resolving the effect of climate change on fish populations. ICES J. Mar. Sci. 66, 1570-

1583. (doi:10.1093/icesjms/fsp056)

551 13. Gilbert, B. et al. 2014 A bioenergetic framework for the temperature dependence of 552 trophic interactions. Ecol. Lett. 17, 902-14. (doi:10.1111/ele.12307)

553 14. O’Connor, M. I., Gilbert, B. \& Brown, C. J. 2011 Theoretical predictions for how 554 temperature affects the dynamics of interacting herbivores and plants. Am. Nat. 178, 626555 38. (doi:10.1086/662171)

556 15. Frances, D. N. \& McCauley, S. J. 2018 Warming drives higher rates of prey consumption 557 and increases rates of intraguild predation. Oecologia 187, 585-596. (doi:10.1007/s00442$558 \quad 018-4146-y)$

559 16. Lurgi, M., López, B. C. \& Montoya, J. M. 2012 Novel communities from climate change.

560 Philos. Trans. R. Soc. B Biol. Sci. 367, 2913-2922. (doi:10.1098/rstb.2012.0238)

561 17. Shurin, J. B., Clasen, J. L., Greig, H. S., Kratina, P. \& Thompson, P. L. 2012 Warming 562 shifts top-down and bottom-up control of pond food web structure and function. Philos. 
Trans. R. Soc. B Biol. Sci. 367, 3008-3017. (doi:10.1098/rstb.2012.0243)

564 18. Yvon-Durocher, G. et al. 2015 Five Years of Experimental Warming Increases the Biodiversity and Productivity of Phytoplankton. PLoS Biol. 13, 1-22. (doi:10.1371/journal.pbio.1002324)

567 19. Ruel, J. J. \& Ayres, M. P. 1999 Jensen's inequality predicts effects of environmental variation. Trends Ecol. Evol. 14, 361-366. (doi:10.1016/S0169-5347(99)01664-X)

569 20. Roitberg, B. D. \& Mangel, M. 2016 Cold snaps, heatwaves, and arthropod growth. Ecol. Entomol. 41, 653-659. (doi:10.1111/een.12324)

571 21. Pörtner, H. O. \& Knust, R. 2007 Climate Change Affects Marine Fishes Through the Oxygen Limitation of Thermal Tolerance. Science (80-. ). 315, 95-97. (doi:10.1126/science.1135471)

574 22. Rideout, R. M., Rose, G. A. \& Burton, M. P. M. 2005 Skipped spawning in female 575 iteroparous fishes. Fish Fish. 6, 50-72.

576 23. Pauly, D. \& Pullin, R. S. V 1988 Hatching time in spherical, pelagic, marine fish eggs in 577 response to temperature and egg size. Environ. Biol. fishes 22, 261-271.

578 24. O’Connor, M. I., Bruno, J. F., Gaines, S. D., Halpern, B. S., Lester, S. E., Kinlan, B. P. \& Weiss, J. M. 2007 Temperature control of larval dispersal and the implications for marine ecology, evolution, and conservation. Proc. Natl. Acad. Sci. U. S. A. 104, 1266-1271.

582 25. Neuheimer, A. B., Thresher, R. E., Lyle, J. M. \& Semmens, J. M. 2011 Tolerance limit for 583 fish growth exceeded by warming waters. Nat. Clim. Chang. 1, 110-113. (doi:doi:10.1038/nclimate1084)

585 26. Deutsch, C. A., Tewksbury, J. J., Huey, R. B., Sheldon, K. S., Ghalambor, C. K., Haak, D. 

latitude. Proc. Natl. Acad. Sci. U. S. A. 105, 6668-6672. (doi:10.1073/pnas.0709472105)

27. Paaijmans, K. P., Heinig, R. L., Seliga, R. a., Blanford, J. I., Blanford, S., Murdock, C. C. \& Thomas, M. B. 2013 Temperature variation makes ectotherms more sensitive to climate change. Glob. Chang. Biol. 19, 2373-2380. (doi:10.1111/gcb.12240)

591 28. Estay, S., Clavijo-Baquet, S., Lima, M. \& Bozinovic, F. 2011 Beyond average: An experimental test of temperature variability on the population dynamics of Tribolium confusum. Popul. Ecol. 53, 53-58. (doi:10.1007/s10144-010-0216-7)

594 29. Pickett, E. J., Thomson, D. L., Li, T. A. \& Xing, S. 2015 Jensen's Inequality and the Impact of Short-Term Environmental Variability on Long-Term Population Growth Rates. PLoS One 10, e0136072. (doi:10.1371/journal.pone.0136072)

30. Bernhardt, J. R., Sunday, J. M., Thompson, P. L. \& O'Connor, M. I. 2018 Nonlinear averaging of thermal experience predicts population growth rates in a thermally variable environment. Proc. R. Soc. B Biol. Sci. 285. (doi:10.1098/rspb.2018.1076)

31. Stillman, J. H. 2019 Heat waves, the new normal: Summertime temperature extremes will impact animals, ecosystems, and human communities. Physiology 34, 86-100.

603 32. Schulte, P. M., Healy, T. M. \& Fangue, N. a. 2011 Thermal performance curves, phenotypic plasticity, and the time scales of temperature exposure. Integr. Comp. Biol. 51,

606 33. Dell, A. I., Pawar, S. \& Savage, V. M. 2011 Systematic variation in the temperature 10591-6. (doi:10.1073/pnas.1015178108) 
609 34. Dell, M., Jones, B. F., Olken, B. A. \& Gates, M. 2014 What Do We Learn from the

$610 \quad$ Weather $\square$ ? The New Climate - Economy Literature. 52, 740-798.

611 35. Ohlberger, J., Edeline, E., Vøllestad, L. A., Stenseth, N. C. \& Claessen, D. 2011

612 Temperature-driven regime shifts in the dynamics of size-structured populations. Am. Nat.

613 177, 211-223. (doi:10.1086/657925)

614 36. Lindmark, M., Huss, M., Ohlberger, J. \& Gårdmark, A. 2018 Temperature-dependent 615 body size effects determine population responses to climate warming. Ecol. Lett. 21, 181616 189. (doi:10.1111/ele.12880)

617 37. Rudolf, V. H. W. \& Roman, A. 2018 Trophic structure alters consequences of 618 environmental warming. Oikos 127, 1646-1656. (doi:10.1111/oik.05535)

619 38. Vázquez, D. P., Gianoli, E., Morris, W. F. \& Bozinovic, F. 2017 Ecological and evolutionary impacts of changing climatic variability. Biol. Rev. 92, 22-42. (doi:10.1111/brv.12216)

39. Post, E. 2013 Ecology of climate change: the importance of biotic interactions. Princeton University Press.

624 40. Vasseur, D. A. \& McCann, K. S. 2005 A mechanistic approach for modeling temperaturedependent consumer-resource dynamics. Am. Nat. 166, 184-198. (doi:10.1086/431285)

626 41. Binzer, A., Guill, C., Rall, B. C. \& Brose, U. 2016 Interactive effects of warming, eutrophication and size structure: Impacts on biodiversity and food-web structure. Glob. Chang. Biol. 22, 220-227. (doi:10.1111/gcb.13086)

629 42. Sentis, A., Binzer, A. \& Boukal, D. S. 2017 Temperature-size responses alter food chain 630 persistence across environmental gradients. Ecol. Lett. 20, 852-862.

$631 \quad$ (doi:10.1111/ele.12779) 
632 43. Fussmann, K. E., Schwarzmüller, F., Brose, U., Jousset, A. \& Rall, B. C. 2014 Ecological 633 stability in response to warming. Nat. Clim. Chang. 4, 206-210. (doi:10.1038/nclimate2134)

635 44. Englund, G., Öhlund, G., Hein, C. L. \& Diehl, S. 2011 Temperature dependence of the functional response. Ecol. Lett. 14, 914-921. (doi:10.1111/j.1461-0248.2011.01661.x)

637 45. Angilletta, M. J. 2006 Estimating and comparing thermal performance curves. J. Therm. Biol. 31, 541-545. (doi:10.1016/j.jtherbio.2006.06.002)

639 46. Amarasekare, P. \& Savage, V. 2012 A framework for elucidating the temperature dependence of fitness. Am. Nat. 179, 178-91. (doi:10.1086/663677)

641 47. Schoolfield,R. M., Sharpe,P. J. \& Magnuson,C. E. 1981 Non-linear regression of biological temperature-dependent rate models based on absolute reaction-rate theory. $J$.

644 48. Padfield, D., Yvon-Durocher, G., Buckling, A., Jennings, S. \& Yvon-Durocher, G. 2016 Rapid evolution of metabolic traits explains thermal adaptation in phytoplankton. Ecol. Lett. 19, 133-142. (doi:10.1111/ele.12545)

647 49. Bernhardt, J. R., Sunday, J. M. \& O’Connor, M. I. 2018 Metabolic Theory and the Temperature-Size Rule Explain the Temperature Dependence of Population Carrying Capacity. Am. Nat. 192, 687-697. (doi:10.1086/700114)

650 50. Sentis, A., Haegeman, B. \& Montoya, J. M. 2019 Stoichiometric constraints modulate the 651 effects of temperature and nutrients on biomass distribution and community stability.

652 bioRxiv , 1-52. (doi:10.1101/589895)

653 51. Fussmann, G. F. \& Heber, G. 2002 Food web complexity and chaotic population 654 dynamics. Ecol. Lett. 5, 394-401. (doi:10.1046/j.1461-0248.2002.00329.x) 
655 52. Pörtner, H. O. \& Farrell, A. P. 2008 ECOLOGY: Physiology and Climate Change.

656 Science (80-. ). 322, 690-692. (doi:10.1126/science.1163156)

657 53. Quinn, T. J. \& Deriso, R. B. 1999 Quantitative fish dynamics. New York: Oxford $658 \quad$ University Press.

659 54. Estay, S., Lima, M. \& Bozinovic, F. 2014 The role of temperature variability on insect 660 performance and population dynamics in a warming world. Oikos 123, 131-140. (doi:10.1111/j.1600-0706.2013.00607.x)

662 55. Bozinovic, F., Bastías, D. a., Boher, F., Clavijo-Baquet, S., Estay, S. A. \& Angilletta, M. 663 J. 2011 The Mean and Variance of Environmental Temperature Interact to Determine 664 Physiological Tolerance and Fitness. Physiol. Biochem. Zool. 84, 543-552. (doi:10.1086/662551)

666 56. Rall, B. Ö. C., Vucic-Pestic, O., Ehnes, R. B., EmmersoN, M. \& Brose, U. 2010

667 Temperature, predator-prey interaction strength and population stability. Glob. Chang. Biol. 16, 2145-2157. (doi:10.1111/j.1365-2486.2009.02124.x)

669 57. Iles, A. C. 2014 Toward predicting community-level effects of climate: Relative 670 temperature scaling of metabolic and ingestion rates. Ecology 95, 2657-2668. (doi:10.1890/13-1342.1)

672 58. Kratina, P., Greig, H. S., Thompson, P. L., Carvalho-Pereira, T. S. A. \& Shurin, J. B. 2012 Warming modifies trophic cascades and eutrophication in experimental freshwater communities. Ecology 93, 1421-1430. (doi:10.1890/11-1595.1)

675 59. Imsland, A. K., Foss, A., Folkvord, A., Stefansson, S. O. \& Jonassen, T. M. 2005 The interrelation between temperature regimes and fish size in juvenile Atlantic cod (Gadus 677 morhua): Effects on growth and feed conversion efficiency. Fish Physiol. Biochem. 31, 
679 60. Dell, A. I., Pawar, S. \& Savage, V. M. 2011 Systematic variation in the temperature 10591-10596. (doi:10.1073/pnas.1015178108)

682 61. Savage, V. M., Gillooly, J. F., Brown, J. H., West, G. B. \& Charnov, E. L. 2004 Effects of body size and temperature on population growth. Am. Nat. 163, 429-441. (doi:10.1086/381872)

62. Uszko, W., Diehl, S., Englund, G. \& Amarasekare, P. 2017 Effects of warming on predator-prey interactions - a resource-based approach and a theoretical synthesis. Ecol. Lett. 20, 513-523. (doi:10.1111/ele.12755)

63. Lindmark, M., Ohlberger, J., Huss, M. \& Gårdmark, A. 2019 Size-based ecological interactions drive food web responses to climate warming. Ecol. Lett. 22, 778-786. (doi:10.1111/ele.13235)

691 64. Van De Wolfshaar, K. E., De Roos, A. M. \& Persson, L. 2008 Population feedback after successful invasion leads to ecological suicide in seasonal environments. Ecology 89,

694 65. Crisci, C., Bensoussan, N., Romano, J. C. \& Garrabou, J. 2011 Temperature anomalies and mortality events in marine communities: Insights on factors behind differential mortality impacts in the NW Mediterranean. PLoS One 6.

698 66. Safaie, A., Silbiger, N. J., McClanahan, T. R., Pawlak, G., Barshis, D. J., Hench, J. L., reduces the risk of coral bleaching. Nat. Commun. 9, 1-12. (doi:10.1038/s41467-018- 
702 67. Temple, G. K. \& Johnston, I. A. 1997 The thermal dependence of fast-start performance

703 in fish. J. Therm. Biol. 22, 391-401. (doi:10.1016/S0306-4565(97)00058-2)

704 68. Grigaltchik, V. S., Ward, A. J. W. \& Seebacher, F. 2012 Thermal acclimation of interactions: Differential responses to temperature change alter predator-prey relationship. Proc. R. Soc. B Biol. Sci. 279, 4058-4064. (doi:10.1098/rspb.2012.1277)

69. Geerts, A. N. et al. 2015 Rapid evolution of thermal tolerance in the water flea Daphnia. 